(DAS28-CRP <2.7) in this study. The CT of metacarpophalangeal (MCP) and proximal interphalangeal (PIP) joints of 2 nd to 5 th fingers was bilaterally visualized and measured at the middle portion of MCP and PIP joints from a longitudinal dorsal view, with approximately 90 degrees flexion. In addition, JSN of finger were scored by van der Heijde- modified Sharp method for patients who had a hand $\mathrm{X}$-ray within 2 months.

Results: CT in MCP joints ranged from 0.0 to $1.1 \mathrm{~mm}$ (median $0.5 \mathrm{~mm}$ ), and CT in PIP ranged from 0.0 to $0.6 \mathrm{~mm}$ (median $0.3 \mathrm{~mm}$ ), respectively. The sum of total CT from 8 fingers ranged from 4.0 to $9.4 \mathrm{~mm}$ (median $6.9 \mathrm{~mm}$ ), and there was a significant difference in total CT, but not in JSN score, between male and female patients (7.4 versus 6.7, $p=0.006$; and 11 versus $10, p=0.899$, respectively). CT was well correlated with JSN $(\rho=-0.589, p<0.001 ; \rho=-0.595$, $p<0.001$ for MCP joints and $\rho=-0.448, p<0.001$ for PIP joints), and both CT and JSN were significantly correlated with disease duration $(\rho=-0.282, p=0.002$, and $\rho=0.286, p=0.005$, respectively). Notably, CT of MCP, but not of PIP, was inversely correlated with disease duration $(\rho=-0.328, p<0.001)$. Height was correlated with CT $(\rho=0.244, p=0.008)$, but not with JSN $(\rho=-0.057, p=0.589)$. CT and JSN were not correlated with age, functional disability, and seropositivity. Conclusions: A direct visualization and quantification of finger joint $\mathrm{CT}$, especially MCP joints, by US is valid and useful in RA.

Disclosure of Interest: None declared

DOI: 10.1136/annrheumdis-2017-eular.4703

\section{SAT0662 EVALUATION OF ANTI-DOUBLE STRANDED DNA ANTIBODIES IN THE MONITORING OF SYSTEMIC LUPUS ERYTHEMATOSUS}

$\underline{\text { T. Dervieux }}^{1}$, T. O'Malley ${ }^{1}$, J. Conklin ${ }^{1}$, C. Ibarra ${ }^{1}$, C. Bentow ${ }^{2}$, M.A. Aure ${ }^{2}$ M. Mahler ${ }^{2} .{ }^{1}$ Exagen Diagnostics, Vista, $C A ;{ }^{2}$ Research and Development, Inova Diagnostics, San Diego, CA, United States

Background: Systemic lupus erythematosus (SLE) is a chronic autoimmune rheumatic disease characterized by the production of pathogenic autoantibodies. Amongst these antibodies, those directed to dsDNA are routinely used to monitor disease activity and are components of the SELENA-SLEDAI index to score severity of SLE disease. Because the predictive value of anti-dsDNA is dependent on the sensitivity and robustness of the assays used, the choice of anti-dsDNA is crucial in the clinical laboratory and clinical research setting.

Objectives: The objective was to compare four anti-dsDNA assays for their performance characteristics of SLE disease activity.

Methods: A cohort of 36 subjects with active SLE presenting with classical complement activation were enrolled and followed monthly for 1 year. At each study visit blood was collected, serum isolated and frozen until analysis. A total of 371 specimens were collected. Disease activity was scored on the day of each study visit according to the SELENA-SLEDAl method excluding anti-dsDNA or complement components (non-serological [ns] SELENA-SLEDAI). All specimens were tested using four different anti-dsDNA kits; QUANTA Lite, QUANTA Flash, a high Avidity anti-dsDNA ELISA, and the Crithidia luciliae indirect immunofluorescence assay (CLIFT) (Inova Diagnostics, San Diego, CA). Study visits presenting with inactive disease (ns-SELENA- SLEDAI score $=0$ ) were compared to those presenting with active disease (ns-SELENA- SLEDAl>0). The longitudinal data were analyzed using linear mixed effect modeling with the ns-SELENA-SLEDAI as dependent variable and the anti-dsDNA titers as fixed effect predictors. Marginal $\mathrm{R}^{2}$ was calculated for each assay.

Results: At enrollment the sensitivity of the QUANTA Lite and High Avidity anti-dsDNA both reached $64 \%$; whereas anti-dsDNA positivity was $83 \%$ by QUANTA Flash and reached $96 \%$ by CLIFT. Study visits with active disease presented with several fold higher anti-dsDNA titers than those with inactive disease status (Table 1). Linear mixed effect modeling indicated that the decrease in ns-SELENA-SLEDAI scores were associated with significant reduction in titers of all three anti-dsDNA kits (Table 2). QUANTA Flash yielded highest marginal R $^{2}$ (0.112) (Table 2).

Table 1. Anti-dsDNA antibody and disease activity

\begin{tabular}{lcccc}
\hline Anti-dsDNA & & Inactive Disease & Active disease & $p$ value \\
& & $0(\mathrm{n}=141)$ & $1+(\mathrm{n}=230)$ & \\
\hline QUANTA Flash & Titers (units) & $72(30-134)$ & $170(56-813)$ & $<0.001$ \\
& Percent positive $(>35$ Units $)$ & $72 \%$ & $86 \%$ & $<0.001$ \\
High Avidity & Titers (units) & $36(12-170)$ & $129(47-775)$ & $<0.001$ \\
& Percent positive $(>30$ Units) & $56 \%$ & $77 \%$ & $<0.001$ \\
QUANTA Lite & Titers (units) & $343(106-624)$ & $545(293-879)$ & $<0.001$ \\
& Percent positive $(>301$ Units) & $53 \%$ & $74 \%$ & $<0.001$ \\
CLIFT & Titers (units) & $1: 160(1: 80-1: 320)$ & $1: 320(1: 160-1: 320)$ & $<0.001$ \\
& Percent positive $(>1: 10)$ & $99 \%$ & $97 \%$ & 0.26
\end{tabular}

Titers are presented as median inter-quartile range.

Table 2. Linear Mixed model effects of anti-dsDNA with ns-SELENA-SLEDAI scores

\begin{tabular}{lccc}
\hline Assay & Intercept + Estimate SE & $p$ value & Marginal $R^{2}$ \\
\hline QUANTA Flash & $3.3 \pm 0.5+0.0011 \pm 0.001$ & $<0.001$ & 0.112 \\
High Avidity & $2.8 \pm 0.8+0.0023 \pm 0.0011$ & 0.001 & 0.082 \\
QUANTA Lite & $2.9 \pm 0.6+0.0037 \pm 0.001$ & 0.022 & 0.037 \\
CLIFT & $3.3 \pm 0.8+0.0031 \pm 0.003$ & 0.28 & 0.006 \\
\hline
\end{tabular}

Conclusions: These preliminary data indicate that anti-dsDNA antibodies determined by QUANTA Flash have value in monitoring SLE disease activity.
Disclosure of Interest: T. Dervieux: None declared, T. O'Malley: None declared, J. Conklin: None declared, C. Ibarra: None declared, C. Bentow Employee of: Inova Diagnostics, M. A. Aure Employee of: Inova Diagnostics, M. Mahler Employee of: Inova Diagnostics

DOI: 10.1136/annrheumdis-2017-eular.5317

\section{SAT0663 TWO-STEP ALGORITHM FOR EARLY DIAGNOSIS OF OSTEOARTHRITIS BASED ON PLASMA DAMAGED AMINO ACIDS PROVIDES SIMPLE AND LOW COST SCREENING TEST}

N. Rabbani, U. Ahmed, P.J. Thornalley. Warwick Medical School, University of Warwick, Coventry, United Kingdom

Background: Currently, magnetic resonance imaging techniques have been developed for evaluation of cartilage damage in early-stage osteoarthritis (eOA). These imaging techniques have approximately $70 \%$ sensitivity and $90 \%$ specificity compared to reference diagnosis by arthroscopy. They require expensive instrumentation, time and facilities. In searching for biomarkers for clinical diagnosis we found that the proteolysis of damaged (oxidised, glycated and nitrated) proteins gives a unique pattern in the plasma of arthritic patients with the severity of the disease - trace level oxidised, glycated and nitrated amino acids. In this study we developed a two-step algorithm using these analytes as features.

Objectives: The objective was to distinguish between the following four groups: healthy control, eOA, early stage rheumatoid arthritis (eRA) and other inflammatory joint disease (non-RA).

Methods: Four algorithm types were tested for performance using random forests, multiclass logistic regression, multi-class sparse logistic regression and support vector machines. In all cases, the diagnostic algorithms were trained on the training data set, before being used to predict the disease class for each sample in the test data set. A two-stage approach was taken: (i) to distinguish between disease and healthy control; and (ii) to distinguish between eOA, eRA and non-RA. The area under the curve of the receiver operating characteristic plot (AUROC) statistic was used as measure of performance

Results: Random forest was the best-performing method. Application of two algorithms consecutively gave the best diagnostic outcome. The AUROC (sensitivity/specificity) values for disease/health were: eOA, $0.99(0.92 / 0.91)$; eRA, $0.96(0.89 / 0.90)$ and non-RA, $0.77(0.73 / 0.72)$ for the training set and test set validations. A random outcome is 0.50 . For typing arthritis, eOA, eRA and non-RA, AUROC values were in the range $0.68-0.98,0.77-0.93$ and $0.62-0.91$ for training set and test set cross-validations and test set validation, respectively. A random outcome is 0.33 .

Conclusions: A two-step algorithm approach based on trace level damaged amino acids gave better diagnostic performance that MRI for detecting and typing eOA. It is low cost and suitable for rollout as a clinical screening test.

References:

[1] Menashe, L., Hirko, K., Losina, E., Kloppenburg, M., Zhang, W., Li, L. and Hunter, D. J. (2012) The diagnostic performance of MRI in osteoarthritis: a systematic review and meta-analysis. Osteoarthritis and Cartilage. 20, 13-21. [2] Ahmed, U., Anwar, A., Savage, R.S., Thornalley, P.J. and Rabbani, N. (2016) Application of protein glycation, oxidation and nitration for early-stage diagnosis and severity of osteoarthritis and other arthritic disease. Arthritis Research \& Therapy 18:250.

Disclosure of Interest: None declared

DOI: 10.1136/annrheumdis-2017-eular.6904

\section{SAT0664 IS THERE AN EARLY ULTRASONOGRAPHIC PATTERN IN SALIVARY GLANDS IN BOTH PRIMARY AND SECONDARY SJOGREN SYNDROME?}

V.C. Iorgoveanu ${ }^{1}$, D. Mazilu ${ }^{2}$, I. Saulescu ${ }^{3}$, V. Vlad ${ }^{4}$, V. Bojinca ${ }^{3}$, L. Groseanu ${ }^{3}$, D. Opris ${ }^{3}$, A. Balanescu ${ }^{3}$, C. Constantinescu ${ }^{3}$, D. Predeteanu ${ }^{3}$, R. Ionescu ${ }^{3}$. ${ }^{1}$ Rheumatology, Carol Davila University of Medicine and Pharmacy; ${ }^{2}$ Saint Mary Clinical Hospital, UMF Carol Davila, Bucharest, Romania; ${ }^{3}$ Rheumatology, Saint Mary Clinical Hospital, UMF Carol Davila; ${ }^{4}$ Rheumatology, Saint Mary Clinical Hospital, Bucharest, Romania

Background: Sjogren Syndrome (SS) affects mainly exocrine glands. Ultrasonography (US) demonstrates specificity and sensibility in major salivary glands (SG) evaluation. Recent data confirm US might be used as primary evaluation technique for its ability to show structural alterations of parenchyma [1].

Objectives: To assess the gray scale (GS) parenchymal inhomogeneity of major SG in patients with established primary and secondary SS and correlate with clinical and biological data.

Methods: Consecutive patients with SS were recruited and SG US was performed. Inhomogeneity of glandular parenchyma was quantified binary on each gland. ESSDAI and ESSPRI scores were calculated. Statistics was performed with SPSS.

Results: Twenty one (42.85\% primary SS, $90.47 \%$ female) consecutive patients were included. Mean age was $53.66+/-12.99$ years and disease duration $5.33+/-$ 3.74 years. Antibody SSA/SSB presence was found in $85.7 \%(18 / 21)$. ESSDAI mean was $8.67+/-8.9(0-29)$, ESSPRI $10.13+/-5.59(0-20)$. There were no differences regarding ESSDAI and ESSPRI in the two groups (primary and secondary SS). Right parotid gland showed alterations in $71.4 \%$ patients $(77 \%$ 